Editorial

\title{
MicroRNAs - A New Generation Molecular Targets for Treating Cellular Diseases
}

\author{
Ramasamy Paulmurugan $\bowtie$ \\ Molecular Imaging Program at Stanford, Bio-X Program, Department of Radiology, Stanford University School of Medicine, Stanford, \\ California, USA \\ $\square$ Corresponding author: Ramasamy Paulmurugan, Ph.D., Department of Radiology, Stanford University School of Medicine, 3155 Porter \\ Drive, \#2236, Palo Alto, CA 94304. Phone: 650-725-6097; Fax: 650-721-6921; Email: paulmur8@stanford.edu \\ ( ) Ivyspring International Publisher. This is an open-access article distributed under the terms of the Creative Commons License (http://creativecommons.org/ \\ licenses/by-nc-nd/3.0/). Reproduction is permitted for personal, noncommercial use, provided that the article is in whole, unmodified, and properly cited.
}

Published: 2013.12 .18

\begin{abstract}
MicroRNAs (miRNAs) are a unique class of non-coding, small RNAs, similar to mRNAs, transcribed by cells, but for entirely different reasons. While mRNAs are transcribed to code for proteins, miRNAs are produced to regulate the production of proteins from mRNAs. miRNAs are central components that tightly and temporally regulating gene expression in cells. Dysregulation of miRNAs expressions in cellular pathogenesis, including cancer, has been reported, and it clearly supports the importance of miRNAs as therapeutic targets in almost every cellular disease. This special issue compiles manuscripts from different authors in order to highlight the importance of microRNAs in cancer and other cellular diseases and how to explore them as therapeutic targets to treat patients with these genetic and metabolic disorders. This issue also covers manuscripts that highlight the obstacles associated with the delivery of therapeutic microRNAs in cells and in vivo in living animals and how to improve and noninvasively monitor the delivery in living animals through molecular imaging, including potential clinical perspectives.
\end{abstract}

Key words: miRNAs, therapeutic targets, cellular disease

MicroRNAs are a novel class of small, non-coding, endogenous RNAs of approximately 18-22 nucleotide long, regulating gene expression post-transcriptionally and controlling major cellular processes, such as cell proliferation, differentiation, and apoptosis [1,2]. Dysregulation or aberrant expression of miRNAs is linked to a variety of cellular pathogenesis, including cancer. One of the major causes of tumorigenesis and subsequent metastasis is reported to be associated with the consequences of dysregulated microRNA expression, which in turn leads to downregulation of various tumor suppressor genes and anti-apoptotic genes and the upregulation of oncogenes $[3,4]$. In the last decade, considerable research has been focused on dissecting the role of miRNAs, the regulatory molecules that are involved in tissue-specific and temporal regulation of human genes, as well as in the initiation, dissemination, and progression of tumorigenesis and metastasis. Comparisons of expression profiles for normal tissues versus human cancers have revealed consistent differences in microRNA expression, especially changes in a specific group of miRNAs in cancerous cells. In some cases, the repression of miRNA maturation promotes tumorigenesis, whereas consistent over-expression of miRNA, such as miR-21, is associated with many forms of cancer. Similarly, over expression of miR-10b and downregulation miR-335 has been linked with invasive and metastatic properties of breast cancer. All these correlations indicate that miRNAs may work either as oncogenes or as tumor suppressors, possibly through post-transcriptional 
regulation of the expression of entire sets of target genes involved in tumorigenesis and metastasis.

MicroRNAs are not only key regulatory agents in cancer; the expression of these regulatory molecules is also significantly altered in various other diseases, such as neurodegenerative disorders (Alzheimer, Parkinson, Schizophrenia, Epilepsy, and Down syndrome), cardiovascular diseases (myocardial Infarction, atherosclerosis, and cardiac fibrosis), and metabolic disorders (diabetes mellitus and obesity). In this special issue, Srinivasan et al. [5] specifically discusses the role of various miRNAs that are dysregulated in major human diseases and how to explore them as therapeutic targets for developing drugs that can either reduce or cure these diseases.

When compared to other diseases, cancer is considered a fatal disease that requires immediate therapeutic strategies. Moreover, microRNAs' roles in cancer have been extensively studied when compare other diseases. In addition, miRNAs have also been explored as a prognostic biomarker in determining the clinical status of the diseases, as well as an indicator for evaluating the therapeutic response of cancers to a given therapy. Although, various imaging modalities, such as CT, MRI, and PET, can noninvasively measure the clinical status of cancer and therapeutic response evaluations in the clinic, the cost and the extensive procedures involved in these modalities are always negative factors; these constraints necessitate the development of a simple and accurate blood-based biomarker test as a substitute for these strategies.

MicroRNAs are emerging as new diagnostic and prognostic molecules, which can be exploited in various aspects. The manuscript presented by Berger and Reiser [6] elaborate on various aspects of miRNAs and their uses in clinical oncology. In addition, they also discuss whether miRNAs can be used as an additional blood-based biomarker that can supplement and improve various imaging results in the clinic or that can be substituted for clinical imaging. To supplement the clinical perspective of miRNAs, Tufman et al. [7] specifically focus on the role of microRNAs in the clinical management of lung cancer, one of the deadliest diseases. Currently available techniques can diagnose lung cancer only at a very late stage, which makes it difficult to treat and improve the patient's life. Lung cancer screening is another complex issue, which takes too much energy and time, with limited success. Taufman et al. [7] discuss, in detail, the complete clinical perspective of lung cancer and how miRNAs can be used to improve, not only the diagnostic aspects of the disease, but also its use as a predictive biomarker in treatment response by the disease.
Inhibition or restoration of miRNAs has been reported to play significant roles in cancer-related events in cells and can be used as promising new generation molecularly targeted anti-cancer therapies. These new strategies are particularly crucial for the treatment of cancers and other diseases that are not responding to conventional treatments and/or may be used as secondary treatments, or in a combination regimen. Therefore, the inhibition or restoration of key miRNAs known to be involved in specific types or stages of cancer, or other pathological status of the cells, may be an effective strategy for the development of novel and targeted therapies to complement conventional treatments. But, the limitations in delivering sense and/or antisense miRNAs, modified to withstand degradation while in circulation, and specifically to target tumor sites in order to achieve significant therapeutic effect are cumbersome. To overcome this issue, our lab [8] and several other groups have been developing polymer nanoparticle (PLA: Poly lacticacid; PLGA: poly(lactic-co-glycolic) acid and PEI: polyethyleneimine) based delivery platforms. The manuscript from our lab details the basics of microRNAs expression, processing, function, and particularly microRNAs' expression that are dysregulated in various cancers. In addition to explaining the importance of microRNA delivery by polymer nanoparticles, we also explained how the delivered microRNAs could be monitored for their functional effects, after their delivery in cells and in living animals by molecular imaging. Furthermore, our manuscript and a manuscript by Yen et al. [9] detail various nanoparticles currently used for drug delivery in cancer therapy, how these nanocarriers are currently being explored for RNAi delivery, and how to use them for microRNA delivery for therapeutic applications and theranostic imaging.

Molecular imaging has been recognized as an important area of bio-medical research, mainly because of its ability to visually represent, characterize, and quantify biological processes in living subjects. For instance, techniques such as Positron Emission Tomography (PET), Single-Photon Emission Computed Tomography (SPECT), Magnetic Resonance Imaging (MRI), and optical imaging (Fluorescence and Bioluminescence) have been extensively used for imaging different biological pathways of cancer cells in small living animals. Molecular imaging uses non-invasive imaging techniques to detect signals that originate from molecules, often in the form of an injected tracers' interaction with a specific cellular target, or a substrate that specifically reacts with enzymes expressed in the target cells, or sometimes from the labeled protein itself, in vivo in living subjects. These techniques are capable of measuring trace 
amounts of radiolabeled probes or optical signals generated from the probes accumulated in the cells or signal emitted by the enzyme-substrate reactions in vivo, and quantifying the molecular kinetic processes that are under review (or being studied). This technique will then provide a wealth of information, facilitating the drug development process, and, thus, enabling therapeutic evaluation and planning. Currently, molecular imaging techniques have been combined with other biochemical assay systems in order to improve early clinical diagnosis of cancers. The importance of molecular imaging techniques is evident in the pharmaceutical industry, starting from drug screening to clinical applications, as well as intermediate processes such as in vitro and pre-clinical evaluations. To highlight the importance molecular imaging and reporter genes applications in microRNA-mediated theranostic imaging we have included a manuscript from $\mathrm{Oh}$ et al. [10], which explains different methods to mimic microRNA function in cells by reporter gene constructs with 3'-UTR regions derived target genes.

Overall, this special issue brings a comprehensive list of information that highlights different aspects of miRNAs, such as function, therapeutic target in cancer and other cellular diseases, the importance of delivery of sense and antisense microRNAs via polymer nanoparticles, and different strategies to monitor their targeted delivery and functions by molecular imaging.

\section{References}

1. Lai EC. Micro RNAs are complementary to $3^{\prime}$ UTR sequence motifs that mediate negative post-transcriptional regulation. Nat Genet. 2002; 30: 363-4.

2. Ambros V. microRNAs: tiny regulators with great potential. Cell. 2001; 107: 823-6.

3. Cimmino A, Calin GA, Fabbri M, Iorio MV, Ferracin M, Shimizu M, et al. miR-15 and miR-16 induce apoptosis by targeting BCL2. Proc Natl Acad Sci U S A. 2005; 102: 13944-9.

4. Wang XF, Shi ZM, Wang XR, Cao L, Wang YY, Zhang JX, et al. MiR-181d acts as a tumor suppressor in glioma by targeting K-ras and Bcl-2. J Cancer Res Clin Oncol. 2012; 138: 573-84.

5. Srinivasan S, Selvan ST, Archunan G, Gulyas B, Padmanabhan P. MicroRNAs the Next Generation Therapeutic Targets in Human Diseases. Theranostics 2013; 3(12):930-942.

6. Berger F, Reiser MF. Micro-RNAs as Potential New Molecular Biomarkers in Oncology: Have They Reached Relevance for the Clinical Imaging Sciences? Theranostics 2013; 3(12):943-952.

7. Tufman A, Tian F, Huber RM. Can MicroRNAs Improve the Management of Lung Cancer Patients? A Clinician's Perspective. Theranostics 2013; 3(12):953-963.

8. Sekar TV, Mohanram RK, Foygel K, Paulmurugan R. Therapeutic Evaluation of microRNAs by Molecular Imaging. Theranostics 2013; 3(12):964-985.

9. Yen SK, Padmanabhan P, Selvan ST. Multifunctional Iron Oxide Nanoparticles for Diagnostics, Therapy and Macromolecule Delivery. Theranostics 2013; 3(12):986-1003.

10. Oh SW, Hwang DW, Lee DS. In vivo Monitoring of microRNA Biogenesis Using Reporter Gene Imaging. Theranostics 2013; 3(12):1004-1011. 